\title{
Behaviour of 4,6-Diaryl-2(1H) pyrimidine-2-thiones Towards Some Electrophiles and Nucleophiles
}

\author{
Nadia T.A. Dawood", Nahed F. Abdel-Ghaffar and Fekria \\ M.A. Soliman \\ Department of Chemistry, Faculty of Science "Girls", Al-Azhar \\ University, Cairo, Egypt.
}

\begin{abstract}
4
,6-DIARY1-1,2-dihydro-2(1H)-pyrimidine-2-thiones $(1 a, b)$ were used for the synthesis of several new pyrimidine derivatives. They were further subjected to hetero ring anellation affording isoxazolo $[4,5-d]$ thiazolo $[2,3-a]$ pyrimidine $(5,6)$ and isoxazolo $[4,5-$ $d]$ thiazino $[2,3-\mathrm{a}]$ pyrimidines $(11,12)$. Biological evaluation of some of the prepared compounds revealed promising antimicrobial activity.
\end{abstract}

Keywords: Pyrimidinethione, Pyrimidothiazine, Thiazolopyrimidine and Antimicrobial activity.

It is well known that pyrimidine and fused pyrimdine heterocycles are of great biological interest, especially as antiviral, antimicrobial ${ }^{(1-8)}$ and antitumor agents ${ }^{(9)}$. It has been $\operatorname{shown}^{(3,10)}$ that some pyrimido-thiazine and thiazolopyrimidine derivatives exhibited marked antibacterial activity against some gram-positive bacteria strains. Also, some thiazolopyrimidines were tested for their antiinflammatory activity and exerted a moderate effect ${ }^{(4)}$. In conjunction with our previous work on the synthesis of pyrimidinethione derivatives for biological evaluations ${ }^{(11-16)}$, we report herein on the synthesis of a series involving the pyrimidine-2-thione moiety and screening of the antimicrobial activity of some of the new derivatives.

\section{Experimental}

All melting points were measured in capillary tubes using an electro-thermal GallenKamp apparatus and are uncorrected. The IR spectra were recorded from $\mathrm{KBr}$ pellets on a Pye Unicam SP 3-300 spectrophotometer. The ${ }^{1} \mathrm{H}-\mathrm{NMR}$ spectra were run on a Varian Gemini NMR spectrometer in deuterated dimethylsulfoxide (DMSO-d6) or deuterated chloroform $\left(\mathrm{CDCl}_{3}\right)$ at $300 \mathrm{MHz}$ using tetramethyl silane (TMS) as internal reference and results are expressed as $\delta$ values ppm. The mass spectra were performed on a Shimadzu GCMS-QP 1000 Ex mass spectrometer at $70 \mathrm{eV}$. The elemental analysis was carried out at the Micoanalytical Center of Cairo University.

Compounds $1 a, 1 b$ have been prepared as previously reported ${ }^{(11)}$.

*E-mail: dawoudnadia@yahoo.com. 
Reduction of 4,6-diaryl-1,2- dihydro pyrimidine-2-thione: Formation of 4,6diaryl-1,2,3,4-tetrahydropyrimidine-2-thiones $(2 a, 2 b)$

To a suspension of compounds $1 a$ or $1 b(0.01 \mathrm{~mol})$ in glacial acetic acid was added stepwise $\mathrm{Zn}$ dust $(0.02 \mathrm{~mol})$ in portions while stirring for half an hour at room temperature. After completion, the reaction mixture was diluted with water, filtered off, washed well with dilute ethanol and recrystallised from ethanol to give compounds $2 a, 2 b$ as white and pale yellow crystals, respectively (Table 1).

Reaction of $2 a, 2 b$ with chloroacetic acid: Formation of 5, 7-diaryl-2,3-dihdro5H-thiazolo[3,2-a]pyrimidin-3-ones $(3 a, 3 b)$

A mixture of compound $2 a$ or $2 b(0.01 \mathrm{~mol})$, chloroacetic acid $(0.01 \mathrm{~mol})$ and freshly fused sodium acetate $(0.05 \mathrm{~mol})$ in glacial acetic acid -acetic anhydride mixture $(30 \mathrm{ml}, 2: 1)$ was refluxed for $4 \mathrm{hr}$. After cooling, the reaction mixture was diluted with water and the product was collected, washed well with water and dilute alcohol then recrystallized from ethanol to give $3 a, 3 b$, respectively (Table 1 ).

Reaction of $3 a, b$ with aromatic aldehydes:Formation of 5, 7-diaryl-2(arylmethylene)-2, 3-dihdro-5H-thiazolo[3,2-a]pyrimidin-3-ones (4a-f)

A mixture of compound $3 a$ or $3 b(0.01 \mathrm{~mol})$, aromatic aldehydes namely, furfural, thiophene-2-aldehyde, isonicotinaldehyde or benzaldehyde $(0.01 \mathrm{~mol})$ and freshly fused sodium acetate $(0.005 \mathrm{~mol})$ in glacial acetic acid-acetic anhydride mixture (30 ml, 2:1) was refluxed for $3 \mathrm{hr}$. After cooling, it was poured into water and the precipitate formed was collected, washed well with water, then dilute ethanol and recrystallized from the proper solvent to give $4 a-4 f$, respectively (Table 1).

Reaction of 4a,d with hydroxylamine hydrochloride :Formation of 3,6,8-triaryl2,3-dihydro-8H-isoxazolo [5 $, 4: 4,5]$ thiazolo [3,2a]pyrimidines $(5 a, 5 b)$

A mixture of compound $4 a$ or $4 d(0.01 \mathrm{~mol})$, hydroxylamine hydrochloride $(0.01 \mathrm{~mol})$ and freshly fused sodium acetate $(0.05 \mathrm{~mol})$ in $30 \mathrm{~mL}$ of glacial acetic acid was refluxed for $6 \mathrm{~h}$. After cooling, it was poured into water and the precipitate formed was collected, washed well with water, then with dilute ethanol and recrystallised from the proper solvent as $5 a, 5 b$, respectively (Table 1).

Reaction of $5 b$ with 2-chloroethyl methyl ether: Formation of 6-(4- bromophenyl)-2(2-methoxyethyl) -8-phenyl-3- (2-thienyl) -2,3-dihydro- $8 H$ - isoxazolo [5 '4 :4,5] thiazolo[2,3-a]pyrimidine (6)

A mixture of compound $5 b(0.01 \mathrm{~mol})$ and sodium hydride $(0.01 \mathrm{~mol})$ in 25 $\mathrm{ml}$ of DMF was stirred on a steam-bath (adjusted at $70^{\circ} \mathrm{C}$ ) for $2 \mathrm{hr}$ and then 2 chloroethyl methyl ether $(0.01 \mathrm{~mol})$ was added. Stirring was continued at $70^{\circ} \mathrm{C}$ for $24 \mathrm{hr}$. The excess solvent was evaporated (reduced pressure) and the product was triturated with light petrol then recrystallized from the proper solvent to give 6 (Table 1). 
TABLE 1.

\begin{tabular}{|c|c|c|c|c|c|c|c|c|c|}
\hline \multirow{2}{*}{$\begin{array}{c}\text { Compd. } \\
\text { No. }\end{array}$} & \multirow{2}{*}{$\begin{array}{l}\text { M.P. } .^{\circ} \mathrm{C} \\
\text { solvent } \\
\text { of cryst. }\end{array}$} & \multirow{2}{*}{$\begin{array}{c}\text { Yield } \\
\%\end{array}$} & \multirow{2}{*}{$\begin{array}{l}\text { Molecular } \\
\text { formula } \\
\text { (Mol.wt.) }\end{array}$} & \multicolumn{3}{|c|}{ Analysis } & \multicolumn{3}{|c|}{ Calcd/found\% } \\
\hline & & & & C & $\mathbf{H}$ & $\mathbf{N}$ & $\mathbf{S}$ & $\mathrm{Cl}$ & $\mathrm{Br}$ \\
\hline \multirow{2}{*}{$2 \mathrm{a}$} & 205.206 & \multirow{2}{*}{65} & $\mathrm{C}_{16} \mathrm{H}_{13} \mathrm{SN}_{2} \mathrm{Cl}$ & 63.89 & 4.32 & 9.31 & 10.64 & 11.81 & \\
\hline & $\mathrm{EtOH}$ & & (300.5) & 63.9 & 4.3 & 9.3 & 10.7 & 11.8 & \\
\hline \multirow{2}{*}{$2 \mathrm{~b}$} & $189-190$ & \multirow{2}{*}{72} & $\mathrm{C}_{16} \mathrm{H}_{13} \mathrm{SN}_{2} \mathrm{Br}$ & 55.65 & 3.76 & 8.11 & 9.27 & & 23.18 \\
\hline & $\mathrm{EtOH}$ & & (345) & 55.7 & 3.8 & 8.1 & 9.3 & & 23.2 \\
\hline \multirow{2}{*}{$3 a$} & $223-224$ & \multirow{2}{*}{60} & $\mathrm{C}_{18} \mathrm{H}_{17} \mathrm{OSN}_{2} \mathrm{Cl}$ & 63.43 & 3.81 & 8.22 & 9.39 & 10.42 & \\
\hline & $\mathrm{EtOH}$ & & (340.5) & 63.4 & 3.8 & 8.2 & 9.4 & 10.4 & \\
\hline \multirow{2}{*}{$3 b$} & $215-216$ & \multirow{2}{*}{65} & $\mathrm{C}_{18} \mathrm{H}_{13} \mathrm{OSN} \mathrm{OSr}_{2} \mathrm{Br}$ & 56.103 & 3.37 & 7.27 & 8.31 & & 20.77 \\
\hline & $\mathrm{EtOH}$ & & (385) & 56.1 & 3.4 & 7.3 & 8.3 & & 20.8 \\
\hline \multirow{2}{*}{$4 a$} & 231-232 & \multirow{2}{*}{64} & $\mathrm{C}_{23} \mathrm{H}_{15} \mathrm{O}_{2} \mathrm{SN}_{2} \mathrm{Cl}$ & 65.94 & 3.58 & 6.69 & 7.64 & 8.48 & \\
\hline & B & & (418.5) & 65.9 & 3.6 & 6.7 & 7.6 & 8.5 & \\
\hline \multirow{2}{*}{$4 b$} & $177-178$ & \multirow{2}{*}{67} & $\mathrm{C}_{23} \mathrm{H}_{15} \mathrm{OS}_{2} \mathrm{~N}_{2} \mathrm{Cl}$ & 63.52 & 3.45 & 6.44 & 14.72 & 8.17 & \\
\hline & B & & (434.5) & 63.5 & 3.5 & 6.4 & 14.7 & 8.2 & \\
\hline \multirow{2}{*}{$4 c$} & $199-200$ & \multirow{2}{*}{62} & $\mathrm{C}_{23} \mathrm{H}_{15} \mathrm{O}_{2} \mathrm{SN}_{2} \mathrm{Br}$ & 59.61 & 3.23 & 6.04 & 6.91 & & 17.27 \\
\hline & B-P.E. & & (463) & 59.6 & 3.2 & 6.1 & 9.6 & & 17.3 \\
\hline \multirow{2}{*}{$4 d$} & $213-214$ & \multirow{2}{*}{69} & $\mathrm{C}_{23} \mathrm{H}_{15} \mathrm{OS}_{2} \mathrm{~N}_{2} \mathrm{Br}$ & 57.62 & 3.13 & 5.84 & 13.36 & & 16.701 \\
\hline & $\mathrm{EtOH}$ & & (479) & 57.5 & 3.1 & 5.8 & 13.4 & & 16.7 \\
\hline \multirow{2}{*}{$4 \mathrm{e}$} & $183-184$ & \multirow{2}{*}{60} & $\mathrm{C}_{24} \mathrm{H}_{16} \mathrm{OSN}_{3} \mathrm{Br}$ & 60.75 & 3.37 & 8.86 & 6.75 & & 16.87 \\
\hline & P.E. & & (474) & 60.8 & 3.4 & 8.9 & 6.8 & & 16.9 \\
\hline \multirow{2}{*}{$4 \mathrm{f}$} & $235-236$ & \multirow{2}{*}{83} & $\mathrm{C}_{25} \mathrm{H}_{17} \mathrm{OSN} \mathrm{OSr}_{2} \mathrm{Br}$ & 63.42 & 3.59 & 5.91 & 6.76 & & 16.91 \\
\hline & $\mathrm{B}$ & & (473) & 63.4 & 3.6 & 5.9 & 7.8 & & 16.9 \\
\hline \multirow{2}{*}{$5 \mathrm{a}$} & $260-261$ & \multirow{2}{*}{60} & $\mathrm{C}_{23} \mathrm{H}_{16} \mathrm{O}_{2} \mathrm{SN}_{3} \mathrm{Cl}$ & 63.66 & 3.69 & 9.68 & 7.38 & 8.18 & \\
\hline & $\mathrm{AcOEt}$ & & $(433.5)$ & 63.7 & 3.7 & 9.7 & 7.4 & 8.2 & \\
\hline $5 \mathrm{~b}$ & $231-232$ & 65 & $\mathrm{C}_{23} \mathrm{H}_{16} \mathrm{OS}_{2} \mathrm{~N}_{3} \mathrm{Br}$ & 55.87 & 3.23 & 8.5202 & 12.95 & & 16.19 \\
\hline & $\mathrm{AcOH}$ & & (494) & 55.9 & 3.2 & 8.5 & 13.0 & & 16.2 \\
\hline 6 & 197-198 & 50 & $\mathrm{C}_{26} \mathrm{H}_{22} \mathrm{O}_{2} \mathrm{~S}_{2} \mathrm{~N}_{3} \mathrm{Br}$ & 56.52 & 3.98 & 7.608 & 11.59 & & 14.49 \\
\hline & P.E. & & (552) & 56.5 & 4.0 & 7.6 & 11.6 & & 14.5 \\
\hline 7 & $286-287$ & 65 & $\mathrm{C}_{19} \mathrm{H}_{19} \mathrm{OSN}_{2} \mathrm{Cl}$ & 63.59 & 5.29 & 7.81 & 8.92 & 9.902 & \\
\hline ra & AcEt & . & $(358.5)$ & 63.6 & 5.3 & 7.8 & 8.9 & 9.9 & \\
\hline $7 \mathrm{~b}$ & $252-253$ & 72 & & 56.57 & 4.71 & 6.94 & 7.94 & & 19.85 \\
\hline & $\mathrm{CHCl}_{3}$ & & & 56.6 & 4.7 & 6.9 & 7.9 & & 19.9 \\
\hline
\end{tabular}




\section{TABLE 1. Cont}

\begin{tabular}{|c|c|c|c|c|c|c|c|c|c|}
\hline \multirow{2}{*}{$\begin{array}{c}\text { Compd. } \\
\text { No. }\end{array}$} & \multirow{2}{*}{$\begin{array}{l}\text { M.P. }{ }^{\circ} \mathrm{C} \\
\text { solvent } \\
\text { of cryst. }\end{array}$} & \multirow{2}{*}{$\begin{array}{c}\text { Yield } \\
\%\end{array}$} & \multirow{2}{*}{$\begin{array}{c}\text { Molecular } \\
\text { formula } \\
\text { (Mol.wt.) }\end{array}$} & \multicolumn{3}{|c|}{ Analysis } & \multicolumn{3}{|c|}{ Calcd/found\% } \\
\hline & & & & C & $\mathbf{H}$ & $\mathbf{N}$ & $\mathbf{S}$ & $\mathrm{Cl}$ & $\mathrm{Br}$ \\
\hline \multirow{2}{*}{$8 \mathrm{~b}$} & $215-216$ & \multirow{2}{*}{80} & $\mathrm{C}_{19} \mathrm{H}_{16} \mathrm{SN}_{3} \mathrm{Br}$ & 57.28 & 4.02 & 10.55 & 8.04 & & 20.100 \\
\hline & EtOH & & (398) & 57.3 & 4.0 & 10.6 & 8.1 & & 20.1 \\
\hline \multirow{3}{*}{$9 \mathrm{a}$} & & \multirow{3}{*}{63} & $\mathrm{C}_{19} \mathrm{H}_{15} \mathrm{OSN}_{2} \mathrm{Cl}$ & 64.31 & 4.23 & 7.89 & 9.02 & 10.01 & \\
\hline & $\mathrm{EtOH}+\mathrm{AcO}$ & & $(354.5)$ & 64.3 & 4.2 & 7.9 & 9.1 & 10.1 & \\
\hline & & & & & & & & & \\
\hline \multirow{2}{*}{$9 b$} & 284-249 & \multirow{2}{*}{70} & $\mathrm{C}_{19} \mathrm{H}_{15} \mathrm{OSN}_{2} \mathrm{Br}$ & 57.14 & 3.76 & 7.01 & 8.02 & & 20.05 \\
\hline & $\begin{array}{c}\mathrm{H} \\
\mathrm{H}\end{array}$ & & (399) & 57.1 & 3.8 & 7.1 & 8.0 & & 20.1 \\
\hline \multirow{2}{*}{$10 \mathrm{a}$} & $284-285$ & \multirow{2}{*}{81} & $\mathrm{C}_{26} \mathrm{H}_{19} \mathrm{OSN}_{2} \mathrm{Cl}$ & 70.50 & 4.29 & 6.32 & 7.23 & 8.02 & \\
\hline & B & & $(442.5)$ & 70.5 & 4.3 & 6.3 & 7.2 & 8.1 & \\
\hline \multirow{2}{*}{$10 \mathrm{~b}$} & $291-292$ & \multirow{2}{*}{79} & $\mathrm{C}_{26} \mathrm{H}_{18} \mathrm{OSN}_{2} \mathrm{Cl}_{2}$ & 65.40 & 3.77 & 5.87 & 6.70 & 14.88 & \\
\hline & B & & (447) & 65.4 & 3.8 & 5.9 & 6.7 & 14.9 & \\
\hline \multirow{2}{*}{$10 \mathrm{c}$} & $188-184$ & \multirow{2}{*}{84} & $\mathrm{C}_{26} \mathrm{H}_{19} \mathrm{OSN}{ }_{2} \mathrm{Br}$ & 64.06 & 3.90 & 5.74 & 6.57 & & 16.42 \\
\hline & B+P.E. & & (487) & 64.1 & 3.9 & 5.8 & 6.6 & & 16.4 \\
\hline \multirow{2}{*}{$10 \mathrm{~d}$} & $213-214$ & \multirow{2}{*}{75} & $\mathrm{C}_{26} \mathrm{H}_{18} \mathrm{OSN}_{2} \mathrm{ClBr}$ & 59.82 & 3.45 & 5.36 & 6.13 & 6.80 & 15.34 \\
\hline & B & & (521.5) & 59.8 & 3.5 & 5.4 & 6.1 & 6.8 & 15.3 \\
\hline \multirow{2}{*}{$11 \mathrm{a}$} & $286-287$ & \multirow{2}{*}{60} & $\mathrm{C}_{26} \mathrm{H}_{20} \mathrm{OSN}{ }_{3} \mathrm{Cl}$ & 68.19 & 4.37 & 9.18 & 6.99 & 7.75 & \\
\hline & B & & (457.5) & 68.2 & 4.4 & 9.2 & 7.0 & 7.8 & \\
\hline \multirow{2}{*}{$11 b$} & $291-292$ & \multirow{2}{*}{52} & $\mathrm{C}_{26} \mathrm{H}_{19} \mathrm{OSN}_{3} \mathrm{ClBr}$ & 58.15 & 3.54 & 7.82 & 5.96 & 6.61 & 14.91 \\
\hline & $\mathrm{D}$ & & $(536.5)$ & 58.2 & 3.5 & 7.8 & 6.0 & 6.6 & 14.9 \\
\hline \multirow{2}{*}{12} & $234-235$ & \multirow{2}{*}{50} & $\mathrm{C}_{29} \mathrm{H}_{26} \mathrm{O}_{2} \mathrm{SN}_{3} \mathrm{Cl}$ & 67.50 & 5.04 & 8.14 & 6.20 & 6.88 & \\
\hline & B+P.E. & & $(515.5)$ & 67.5 & 5.1 & 8.1 & 6.2 & 6.9 & \\
\hline
\end{tabular}

where : $\mathrm{B}=$ benzene; P.E. $=$ petroleum ether b.p. $80-120^{\circ}$; AcOEt= ethyl acetate and $\mathrm{D}=$ dioxane.

Reaction of 4,6-diaryl -1,2,3,4-tetrahydropyrimidine-2-thiones $2 a, 2 b$ with 2 chloroethylmethylether :Formation of 2,4-diaryl-6-(2-methoxyethyl)sulphanyl1,2-dihydropyrimidine $(7 a, 7 b)$

A mixture of compound $2 a$ and/or $2 b(0.01 \mathrm{~mol}), 2$-chloroethyl methyl ether $(0.01 \mathrm{~mol})$ in ethanol $(50 \mathrm{ml})$ containing sodium hydroxide $(0.0125 \mathrm{~mol})$ was stirred at $60^{\circ} \mathrm{C}$ for $6 \mathrm{hr}$. It was then poured into water $(100 \mathrm{~mL})$ and the precipitate that separated was collected washed well with water and dilute alcohol then recrystallized from ethanol to give $7 a, 7 b$, respectively (Table 1).

Egypt. J. Chem. 54, No.1 (2011) 
Reaction of 2a,b with acrylonitrile: Formation of 3-[4-(4-substituted-phenyl)-2phenyl-6-sulphanyl-1,2-dihydro-1-pyrimidine]propanenitrile $(8 a, 8 b)$

A mixture of compound $2 a$ or $2 b(0.01 \mathrm{~mol})$ and acrylonitrile $(0.06 \mathrm{~mol}, 3 \mathrm{ml})$ in $50 \mathrm{~mL}$ of pyridine was refluxed for $6 \mathrm{hr}$. It was cooled, poured into ice-dilute $\mathrm{HCl}$ and the product was filtered off, washed well with water and recrystallized from ethanol to give $8 a, b$ (Table 1).

Reaction of 3a,b with $\mathrm{AcOH}$ - $\mathrm{HCl}$ mixture. Formation of 6,8-diaryl-3,4-dihydro2H,6H-pyrimido[2,1-b][1,3]-thiazin-2-ones $(9 a, 9 b)$

A suspension of compound $8 a$ or $8 b(0.01 \mathrm{~mol})$ in glacial acetic acidconcentrated hydrochloric acid mixture (by volume) (30-10 ml) was refluxed for $4 \mathrm{hr}$. The reaction mixture was concentrated (reduced pressure) and the semisolid that separated was washed well with water then dried and triturated with light petroleum ether then recrystallized from ethanol acetic acid mixture to give compounds $9 a, 9 b$, respectively (Table 1 ).

Condensation of $9 a, b$ with aromatic aldehydes: Formation of 3-(arylmethylene)6,8-diaryl-3,4-dihydro-2H,6Hpyrimido[2,1-b][1,3]-thiazin-2-ones (10a-10d)

A mixture of compounds $9 a$ or $9 b(0.01 \mathrm{~mol})$, aromatic aldehydes namely, benzaldehyde and/or 2-chlorobenzaldehyde $(0.01 \mathrm{~mol})$ in glacial acetic acidacetic anhydride mixture $(25: 15 \mathrm{ml})$ and freshly fused sodium acetate $(0.05 \mathrm{~mol})$ was heated on a steam bath under reflux for $3 \mathrm{hr}$. After cooling, the reaction mixture was diluted with water and the product was filtered off, washed well with water then with dilute ethanol and recrystallized from the proper solvent to give $10 a-10 d$, (Table 1).

Reaction of 5a,d with hydroxylamine hydrochloride: Formation of 3,6,8-triaryl2,3-dihydro-4H,6H-isoxazolo[4,5-e]pyrimido[2,1-b]thiazine (11a,11b)

A mixture of compound $10 a$ or $10 d(0.01 \mathrm{~mol})$, hydroxylamine hydrochloride $(0.01 \mathrm{~mol})$ in glacial acetic acid $(20 \mathrm{ml})$ was refluxed for $6 \mathrm{hr}$. After cooling it was poured into water and the solid that separated was collected, washed well with dilute alkali $\left(\mathrm{Na}_{2} \mathrm{CO}_{3}\right)$ and recrystallized from benzene or dioxane to give $11 a, 11 b$ (Table 1).

Reaction of 11 a with 2-chloroethyl methyl ether: Formation of 3,6,8-triaryl-2-(2methoxyethyl)-2,3-dihydro-4H,6H-isoxazolo[4,5-e]pyrimido[2,1-b] [1,3]thiazine (12)

To a suspension of $11 a(0.01 \mathrm{~mol})$ in dry DMF $(25 \mathrm{ml})$ was added sodium hydride $(0.4 \mathrm{~g})$ in portions while stirring on a steam-bath (and the temperature was adjusted so not to exceed $65^{\circ} \mathrm{C}$ for $1 \mathrm{hr}$, then 2-chloroethyl methyl ether was added, while stirring, and the reaction mixture was stirred for another $6 \mathrm{hr}$ at $40^{\circ} \mathrm{C}$. The excess solvent was evaporated (reduced pressure) and the semi-solid formed was triturated with light petroleum ether (b.p. 40-60 ) and the product was recrystallized from benzene/pet-ether mixture (b.p. $40-60^{\circ}$ ) to give 12 (Table 1). 
Nadia T.A. Dawood et al.

TABLE 2. Spectral data of the prepared compounds (2-12).

\begin{tabular}{|c|c|c|c|c|}
\hline $\begin{array}{l}\text { Compd. } \\
\text { No. }\end{array}$ & IR spectra ${ }^{a}$ & ${ }^{1} \mathrm{H}-\mathrm{NMR}$ spectrab & $\begin{array}{c}{ }^{13} \mathrm{C} \text {-NMR spectra } \delta \\
(75.5 \mathrm{MHz} \text {, DMSO- } \\
\left.\mathrm{d}_{6}\right) / \mathrm{or} \mathrm{CDCl}_{3}\end{array}$ & $\begin{array}{c}\text { Mass spectra } \\
\mathbf{m} / \mathbf{z}(\%)\end{array}$ \\
\hline $2 a$ & $\begin{array}{l}3330(\mathrm{vNH}) ; \\
2982,2890 \\
(\mathrm{vCH}) ; 2624 \\
((\mathrm{vSH}), 1605 \\
(\mathrm{vC}=\mathrm{N})\end{array}$ & $\begin{array}{l}10.9(\mathrm{~s}, 1 \mathrm{H}, \mathrm{NH}, \\
\mathrm{D}_{2} \mathrm{O} \text { exchangeable), } \\
10.53(\mathrm{~d}, 1 \mathrm{H}, \mathrm{NH}), \\
3.14\left(\mathrm{~d}, 1 \mathrm{H}, \mathrm{C}_{4}-\mathrm{H}\right), \\
4.23\left(\mathrm{~d}, 1 \mathrm{H}, \mathrm{C}_{5}-\mathrm{H}\right), \\
6.79-8.01(\mathrm{~m}, 9 \mathrm{H}, \\
\mathrm{Ar}-\mathrm{H}) .\end{array}$ & $\begin{array}{l}\text { 130.3(CH), } 134.8(\mathrm{CH}), \\
139.4(\mathrm{C}), 185.9(\mathrm{CS}), \\
186.7(\mathrm{CCl}) .\end{array}$ & $\begin{array}{rr}303 & \mathrm{M}+2 \\
& (79.2) \\
301 & \mathrm{M}\end{array}$ \\
\hline 2b & $\begin{array}{l}3290(\mathrm{vNH}) ; \\
3005,2990 \\
(\mathrm{vCH}) ; 2604 \\
((\mathrm{vSH}), 1590 \\
(\mathrm{vC}=\mathrm{N}) .\end{array}$ & $\begin{array}{l}10.91(\mathrm{~s}, 1 \mathrm{H}, \mathrm{NH}, \\
\text { exchangeable), } \\
11.04(\mathrm{~d}, 1 \mathrm{H}, \mathrm{NH} \\
\text { exchangeable), } 4.01 \\
\left(\mathrm{~d}, 1 \mathrm{H}, \mathrm{C}_{4}-\mathrm{H}\right), 4.23 \\
\left(\mathrm{~d}, 1 \mathrm{H}, \mathrm{C}_{5}-\mathrm{H}\right), 6.81- \\
8.12(\mathrm{~m}, 9 \mathrm{H}, \mathrm{Ar}-\mathrm{H}) .\end{array}$ & $\begin{array}{l}\text { 130.4(CH), } 135.6(\mathrm{CH}), \\
\text { 140.6(C), 187.6 (CS), } \\
\text { 189.8(CBr). }\end{array}$ & 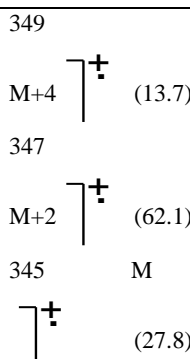 \\
\hline $3 \mathbf{a}$ & $\begin{array}{l}2992,2880 \\
(\mathrm{vCH}) ; 1701, \\
(\mathrm{vC}=\mathrm{O}) ; 1605 \\
(\mathrm{vC}=\mathrm{N})\end{array}$ & $\begin{array}{l}3.45\left(\mathrm{~s}, 2 \mathrm{H}, \mathrm{CH}_{2}\right), \\
3.8\left(\mathrm{~d}, 1 \mathrm{H}, \mathrm{C}_{5}-\mathrm{H}\right), \\
4.1\left(\mathrm{~d}, \mathrm{C}_{6}-\mathrm{H}\right), 7.1- \\
7.92(\mathrm{~m}, 9 \mathrm{H}, \mathrm{Ar}-\mathrm{H}) .\end{array}$ & $\begin{array}{l}\text { 24.6( }\left(\mathrm{CH}_{2}\right), 129.4(\mathrm{CH}), \\
131.2(\mathrm{CH}), 137.4(\mathrm{C}), \\
162.6(\mathrm{CO}), 1606.8 . \\
(\mathrm{CS})\end{array}$ & $\begin{array}{lll}443 & & \\
\mathrm{M}+4 & 7^{+} & \\
341 & & \\
& & \text { M } \\
& & (33.1) \\
& \end{array}$ \\
\hline 3b & $\begin{array}{l}3005,2992, \\
2829(v C H) ; \\
1701,(v C=\mathrm{O}) ; \\
1615(v \mathrm{C}=\mathrm{N})\end{array}$ & $\begin{array}{l}4.01\left(\mathrm{~s}, 2 \mathrm{H}, \mathrm{CH}_{2}\right), \\
4.5\left(\mathrm{~d}, 1 \mathrm{H}, \mathrm{C}_{5}-\mathrm{H}\right), \\
5.3\left(\mathrm{~d}, \mathrm{C}_{6}-\mathrm{H}\right), 7.12- \\
7.95(\mathrm{~m}, 9 \mathrm{H}, \mathrm{Ar}-\mathrm{H}) .\end{array}$ & $\begin{array}{l}\text { 26.4( }\left(\mathrm{CH}_{2}\right), 130(\mathrm{CH}), \\
\text { 131.2(CH), } 137.4(\mathrm{C}), \\
165.4(\mathrm{CO}), 160.2(\mathrm{CS})\end{array}$ & $\begin{array}{rr}389 & \mathrm{M}+4 \\
& (21.5 \\
387 & \mathrm{M}+2 \\
& (10.6) \\
385 & \mathrm{M} \\
& (29.8)\end{array}$ \\
\hline $4 \mathbf{a}$ & $\begin{array}{l}2990,2890 \\
(v \mathrm{CH}) ; 1705 \\
(v \mathrm{C}=\mathrm{O})\end{array}$ & $\begin{array}{l}4.56\left(\mathrm{~d}, 1 \mathrm{H}, \mathrm{C}_{5}-\mathrm{H}\right), \\
5.63\left(\mathrm{~d}, 1 \mathrm{H}, \mathrm{C}_{6} \mathrm{H}\right), \\
7.2-7.96(\mathrm{~m} 13 \mathrm{H}, \\
\text { Ar-H an aryl-CH). }\end{array}$ & $\begin{array}{l}124.3(\mathrm{C}), 126(\mathrm{CH}), \\
129.6(\mathrm{CH}), 130.4(\mathrm{CH}), \\
132.5(\mathrm{CH}) 133.9(\mathrm{CH}), \\
134.3(\mathrm{CH}), 137.4(\mathrm{C}), \\
141.5(\mathrm{C}), 161.2(\mathrm{CO}), \\
165.4(\mathrm{CO}), 169.6(\mathrm{CS})\end{array}$ & $\left.\begin{array}{rr}421 & \mathrm{M}+2 \\
& \\
419 & \mathrm{M}]\end{array}\right]^{-1}$ \\
\hline
\end{tabular}

Egypt. J. Chem. 54, No.1 (2011) 


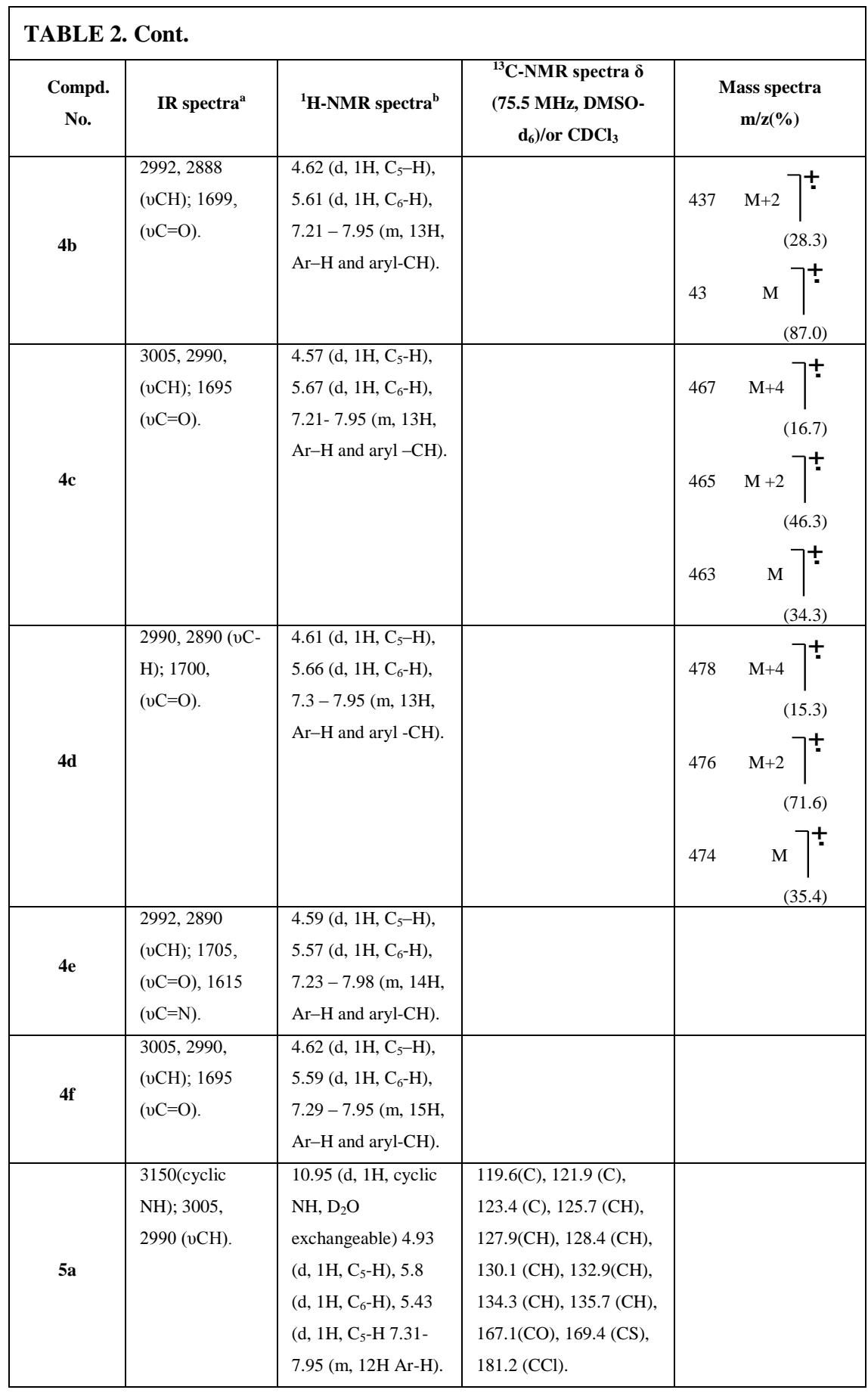




\begin{tabular}{|c|c|c|c|c|}
\hline $\begin{array}{c}\text { Compd. } \\
\text { No. }\end{array}$ & IR spectra ${ }^{a}$ & ${ }^{1}$ H-NMR spectra ${ }^{b}$ & $\begin{array}{c}{ }^{13} \mathrm{C}-\mathrm{NMR} \text { spectra } \delta \\
\left(75.5 \mathrm{MHz}^{\mathrm{DMSO}-\mathrm{d}_{6}}\right) / \\
\text { or } \mathrm{CDCl}_{3}\end{array}$ & $\begin{array}{c}\text { Mass spectra } \\
\mathbf{m} / \mathbf{z}(\%)\end{array}$ \\
\hline $5 b$ & $\begin{array}{l}3235 \text { (cyclic } \\
\mathrm{NH}) ; 2992, \\
2888(v \mathrm{CH}) .\end{array}$ & $\begin{array}{l}10.92(\mathrm{~d}, 1 \mathrm{H}, \text { cyclic } \\
\mathrm{NH}, \mathrm{D}_{2} \mathrm{O} \\
\text { exchangeable }), 4.79 \\
\left(\mathrm{~d}, 1 \mathrm{H}, \mathrm{C}_{9}-\mathrm{H}\right), 5.41 \\
\left(\mathrm{~d}, 1 \mathrm{H}, \mathrm{C}_{5}-\mathrm{H}\right) \\
5.79\left(\mathrm{~d}, 1 \mathrm{H}, \mathrm{C}_{6}-\mathrm{H}\right), \\
7.23-7.89(\mathrm{~m}, 12 \mathrm{H} \\
\mathrm{Ar}-\mathrm{H}) .\end{array}$ & & \\
\hline 6 & $\begin{array}{l}2995,2882, \\
2820(\mathrm{vCH}) .\end{array}$ & $\begin{array}{l}2.10\left(\mathrm{~s}, 3 \mathrm{H}, \mathrm{O}-\mathrm{CH}_{3}\right), \\
2.71\left(\mathrm{~d}, 2 \mathrm{H}, \mathrm{CH}_{2-}\right. \\
\left.\mathrm{CH}_{2}\right), 3.1(\mathrm{t}, 2 \mathrm{H}, \\
\left.\mathrm{CH}_{2} \mathrm{CH}_{2} \mathrm{O}\right), 4.71(\mathrm{~d}, \\
\left.1 \mathrm{H}, \mathrm{C}_{9}-\mathrm{H}\right), 5.13(\mathrm{~d}, \\
\left.1 \mathrm{H}, \mathrm{C}_{4}-\mathrm{H}\right), 5.69(\mathrm{~d}, \\
\left.1 \mathrm{H}, \mathrm{C}_{5}-\mathrm{H}\right), 7.29-7.91 \\
(\mathrm{~m}, 12 \mathrm{H}, \mathrm{Ar}-\mathrm{H}) .\end{array}$ & $\begin{array}{l}\left.\text { 17.3( } \mathrm{CH}_{3}\right), 19.9\left(\mathrm{CH}_{2}\right), \\
22.5\left(\mathrm{CH}_{2} \mathrm{O}\right), 124.3(\mathrm{C}), \\
126.7(\mathrm{C}), 129.6(\mathrm{CH}), \\
130.4(\mathrm{CH}), 132.5(\mathrm{CH}), \\
133.9(\mathrm{CH}), 136.2(\mathrm{C}), \\
137.4(\mathrm{C}), 138(\mathrm{CH}), \\
141.5(\mathrm{C}), 162.7(\mathrm{CO}), \\
168.9(\mathrm{CS})\end{array}$ & 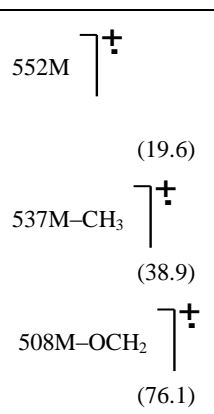 \\
\hline $7 \mathbf{a}$ & $\begin{array}{l}3225(\mathrm{vNH}) \\
3005 ; 2992, \\
2828(\mathrm{vCH}) .\end{array}$ & $\begin{array}{l}10.91(\mathrm{~d}, 1 \mathrm{H}, \mathrm{NH}, \\
\mathrm{D}_{2} \mathrm{O} \text { exchangeable), } \\
2.69(\mathrm{t}, 2 \mathrm{H}, \\
\left.\mathrm{CH}_{2}, \mathrm{CH}_{2}\right), 3.12(\mathrm{t}, \\
\left.2 \mathrm{H}, \mathrm{CH}_{2} \mathrm{O}\right), 3.41,(\mathrm{~s}, \\
\left.3 \mathrm{H}, \mathrm{OCH}_{3}\right), 4.79(\mathrm{~d}, \\
\left.1 \mathrm{H}, \mathrm{C}_{4}-\mathrm{H}\right), 5.69(\mathrm{~m}, \\
\left.1 \mathrm{H}, \mathrm{C}_{5}-\mathrm{H}\right), 7.45-8.01 \\
(\mathrm{~m}, 9 \mathrm{H}, \mathrm{Ar}-\mathrm{H}) .\end{array}$ & $\begin{array}{l}26.4\left(\mathrm{SCH}_{2}\right), 33.1 \\
\left(\mathrm{CH}_{2} \mathrm{O}\right), 79.9\left(\mathrm{OCH}_{3}\right), \\
118.7(\mathrm{CH}), 129.4(\mathrm{C}), \\
129.6(\mathrm{CH}), 130.4(\mathrm{CH}), \\
131.2(\mathrm{C}), 132.8(\mathrm{CH}), \\
133.9(\mathrm{CH}), 136.2(\mathrm{C}), \\
137.4(\mathrm{C}), 169.8(\mathrm{CS})\end{array}$ & \\
\hline $7 \mathrm{~b}$ & $\begin{array}{l}3350(\mathrm{vNH}) ; \\
3005,2990, \\
2881,2829 \\
(\mathrm{vCH}) ; 1605 \\
(\mathrm{vC}=\mathrm{N}) .\end{array}$ & $\begin{array}{l}1.01(\mathrm{~d}, 1 \mathrm{H}, \mathrm{NH}, \\
\mathrm{D}_{2} \mathrm{O} \text { exchangeable), } \\
2.91(\mathrm{t}, 2 \mathrm{H}, \\
\left.\mathrm{CH}_{2}, \mathrm{CH}_{2}\right), 3.14(\mathrm{t}, \\
\left.2 \mathrm{H}, \mathrm{CH}_{2} \mathrm{O}\right), 3.44,(\mathrm{~S}, \\
\left.3 \mathrm{H}, \mathrm{OCH}_{3}\right), 4.95(\mathrm{~d}, \\
\left.1 \mathrm{H}, \mathrm{C}_{4}-\mathrm{H}\right), 5.71(\mathrm{~m}, \\
\left.1 \mathrm{H}, \mathrm{C}_{5}-\mathrm{H}\right), 7.39-7.99 \\
(\mathrm{~m}, 9 \mathrm{H}, \mathrm{Ar}-\mathrm{H}) .\end{array}$ & 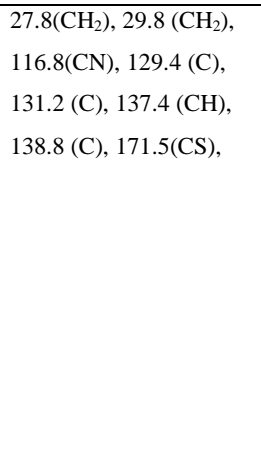 & 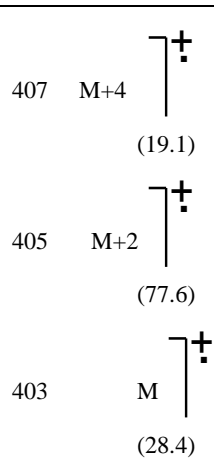 \\
\hline
\end{tabular}

Egypt. J. Chem. 54, No.1 (2011) 


\begin{tabular}{|c|c|c|c|c|}
\hline $\begin{array}{c}\text { Compd. } \\
\text { No. }\end{array}$ & IR spectra ${ }^{a}$ & ${ }^{1} H-N M R$ spectra ${ }^{b}$ & $\begin{array}{c}{ }^{13} \mathrm{C}-\mathrm{NMR} \text { spectra } \delta \\
\left(75.5 \mathrm{MHz}^{\mathrm{MHSO}-\mathrm{d}_{6}}\right) / \\
\text { or } \mathrm{CDCl}_{3}\end{array}$ & $\begin{array}{c}\text { Mass spectra } \\
\mathbf{m} / \mathbf{z}(\%)\end{array}$ \\
\hline $8 a$ & $\begin{array}{l}2223(v \mathrm{C}-\mathrm{N}) \\
2992,2828 \\
(\mathrm{vCH}), 2607 \\
(\mathrm{vSH}) 1595 \\
(\mathrm{vC}=\mathrm{N})\end{array}$ & $\begin{array}{l}8.1\left(\mathrm{~s}, 1 \mathrm{H}, \mathrm{SH}, \mathrm{D}_{2} \mathrm{O}\right. \\
\text { exchangeable), } 2.89 \\
\left(\mathrm{t}, 2 \mathrm{H}, \mathrm{CH}_{2}, \mathrm{CN}\right), \\
3.7\left(\mathrm{t}, 2 \mathrm{H}, \underline{\mathrm{CH}}_{2} \mathrm{CH}_{2}\right), \\
4.94,\left(\mathrm{~d}, 1 \mathrm{H}, \mathrm{C}_{6}-\mathrm{H}\right), \\
5.01\left(\mathrm{~d}, 1 \mathrm{H}, \mathrm{C}_{5}-\mathrm{H}\right), \\
7.1-7.9(\mathrm{~m}, 9 \mathrm{H}, \mathrm{Ar}- \\
\mathrm{H}) .\end{array}$ & $\begin{array}{l}\text { 22.4( }\left(\mathrm{CH}_{2}\right), 27.3\left(\mathrm{CH}_{2}\right), \\
\text { 116.1(CN), } 131.1(\mathrm{C}), \\
\text { 134.5 (CH), } 136.7(\mathrm{CH}), \\
\text { 171.1(CSH). }\end{array}$ & 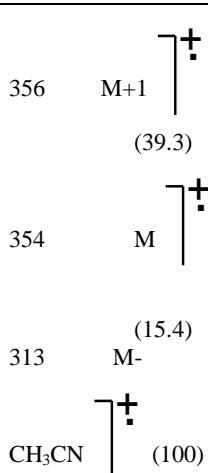 \\
\hline $8 b$ & $\begin{array}{l}2221(v \mathrm{C}-\mathrm{N}), \\
3001,2991, \\
2882,2820 \\
(v \mathrm{CH}), 2627 \\
(\mathrm{vSH}), 1601 \\
(\mathrm{vC}=\mathrm{N}) .\end{array}$ & $\begin{array}{l}8.29\left(\mathrm{~s}, 1 \mathrm{H}, \mathrm{SH}, \mathrm{D}_{2} \mathrm{O}\right. \\
\text { exchangeable), } 2.91 \\
\left(\mathrm{t}, 2 \mathrm{H}, \underline{\mathrm{CH}}_{2}, \mathrm{CN}\right), \\
3.7\left(\mathrm{t}, 2 \mathrm{H}, 2 \mathrm{H}, \underline{\mathrm{CH}}_{2}\right. \\
\left.\mathrm{CH}_{2}\right), 5.1\left(\mathrm{~d}, 1 \mathrm{H}, \mathrm{C}_{6^{-}}\right. \\
\mathrm{H}), 5.31\left(\mathrm{~d}, 1 \mathrm{H}, \mathrm{C}_{5^{-}}\right. \\
\mathrm{H}), 7.3-7.95(\mathrm{~m}, 9 \mathrm{H}, \\
\mathrm{Ar}-\mathrm{H}) .\end{array}$ & & \\
\hline $9 a$ & $\begin{array}{l}2929,2882, \\
2828(\mathrm{vCH}), \\
1699(\mathrm{vC}=\mathrm{O}) .\end{array}$ & $\begin{array}{l}2.59\left(\mathrm{t}, 2 \mathrm{H}, \mathrm{CH}_{2^{-}}\right. \\
\left.\mathrm{CH}_{2}\right), 3.01(\mathrm{t}, 2 \mathrm{H}, \\
\left.2 \mathrm{H}, \mathrm{CH}_{2} \mathrm{CO}\right), 4.95 \\
\left(\mathrm{~d}, 1 \mathrm{H}, \mathrm{C}_{6}-\mathrm{H}\right), 5.41 \\
\left(\mathrm{~d}, 1 \mathrm{H}, \mathrm{C}_{7}-\mathrm{H}\right), 7.12- \\
7.81(\mathrm{~m}, 9 \mathrm{H}, \mathrm{Ar}-\mathrm{H}) .\end{array}$ & $\begin{array}{l}\text { 26.7 }\left(\mathrm{CH}_{2}\right), 27.1\left(\mathrm{CH}_{2}\right), \\
116.8(\mathrm{CN}), 129.4(\mathrm{C}), \\
166.9(\mathrm{CO}), 169.8(\mathrm{CS}) .\end{array}$ & 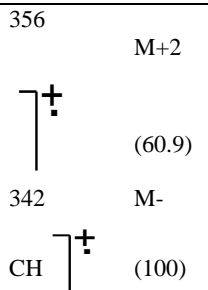 \\
\hline $9 b$ & $\begin{array}{l}3005,2999, \\
2821(v \mathrm{CH}), \\
1701(\mathrm{vC}=\mathrm{O}) .\end{array}$ & $\begin{array}{l}2.61\left(\mathrm{t}, 2 \mathrm{H}, \mathrm{CH}_{2^{-}}\right. \\
\left.\mathrm{CH}_{2}\right), 3.11(\mathrm{t}, 2 \mathrm{H}, \\
\left.\mathrm{CH}_{2} \mathrm{CO}\right), 5.01(\mathrm{~d}, \\
\left.1 \mathrm{H}, \mathrm{C}_{6}-\mathrm{H}\right), 5.65(\mathrm{~d}, \\
\left.1 \mathrm{H}, \mathrm{C}_{7}-\mathrm{H}\right), 7.21-7.83 \\
(\mathrm{~m}, 9 \mathrm{H}, \mathrm{Ar}-\mathrm{H}) .\end{array}$ & & \\
\hline $10 \mathrm{a}$ & $\begin{array}{l}2990,2882, \\
2820(v \mathrm{CH}), \\
1690(v \mathrm{C}=\mathrm{O}) .\end{array}$ & $\begin{array}{l}3.21\left(\mathrm{~S}, 2 \mathrm{H}, \mathrm{CH}_{2}\right), \\
4.91\left(\mathrm{~d}, 1 \mathrm{H}, \mathrm{C}_{6} \mathrm{H}\right), \\
5.3\left(\mathrm{~d}, 1 \mathrm{H}, \mathrm{C}_{7}-\mathrm{H}\right) \\
7.19-8.01(\mathrm{~m}, 15 \mathrm{H}, \\
\mathrm{Ar}-\mathrm{H}) \text { and } 1 \mathrm{H}, \mathrm{Ar}- \\
\mathrm{CH}=\mathrm{C}) .\end{array}$ & $\begin{array}{l}\text { 27.4( }\left(\mathrm{CH}_{2}\right), 114.3(\mathrm{CN}), \\
\text { 129.6(CH), 131.4 (CH), } \\
\text { 132.9(CH), 134.4 (C), } \\
\text { 136.9(C), 137.4 (C), } \\
\text { 161.2(CO), 169.8 (CS). }\end{array}$ & $\begin{array}{l}\text { M+1 } \\
\text { (19.2) } \\
\mathrm{M}\rceil^{+} \\
(22.4)\end{array}$ \\
\hline
\end{tabular}

Egypt. J. Chem. 54, No.1 (2011) 
Nadia T.A. Dawood et al.

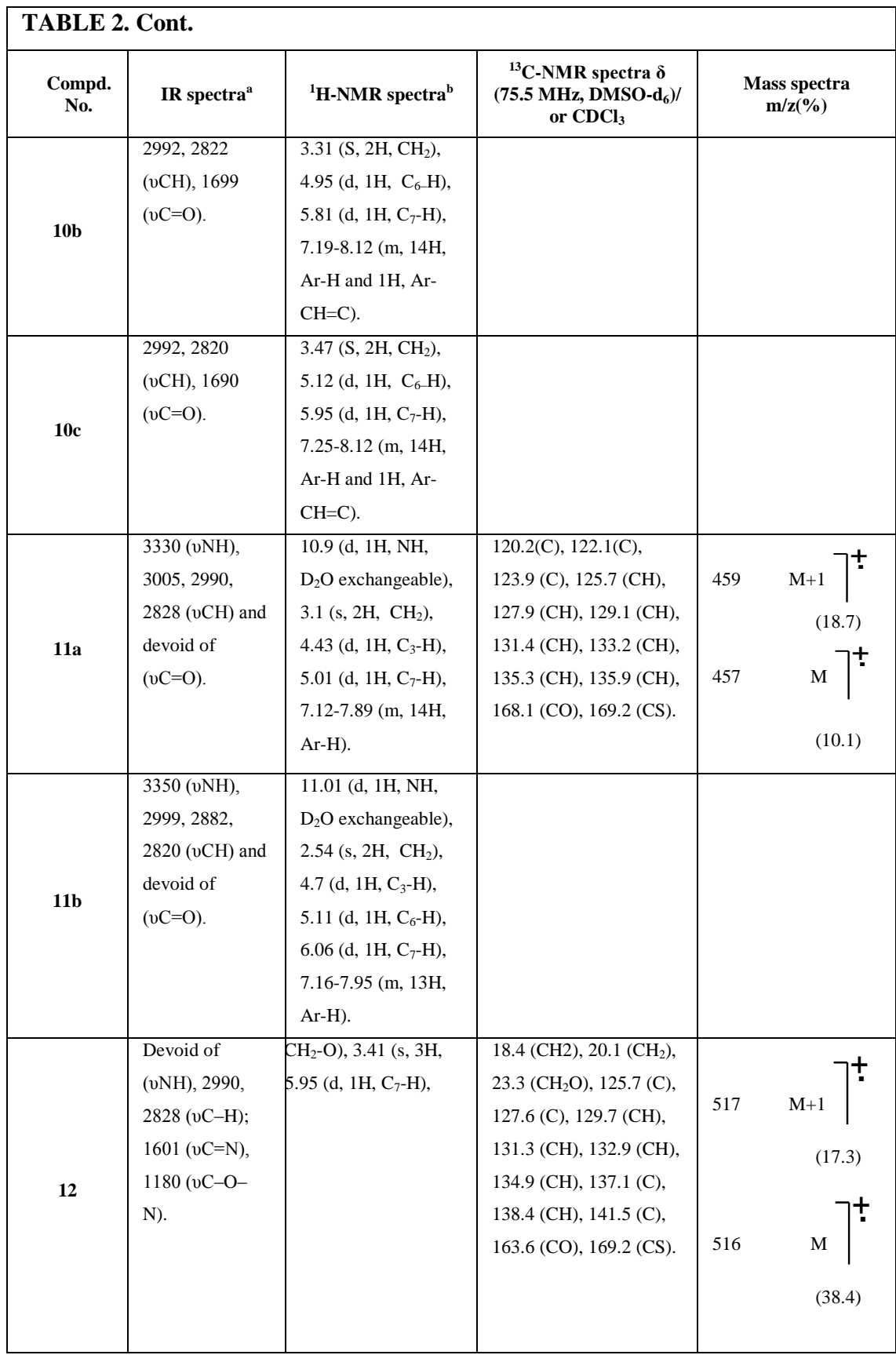

Where a), $v$ in $\mathrm{cm}^{-1} \quad$; b) $\delta$ in ppm

Egypt. J. Chem. 54, No.1 (2011) 


\section{Results and Discussion}

4, 6-Diaryl -1, 2- dihydropyrimidine-2(1H)-thione $(1)^{(11)}$ is used as a key starting compound in the synthesis of fused pyrimidine derivatives. Thus, compounds $1 a, 1 b$ reacted with zinc dust in the presence of glacial acetic acid to give the corresponding 4, 6-diaryl-1, 2, 3, 4-tetrahydropyrimidine-2-thiones $2 a$, $2 b$. The spectral data of compounds 2 agreed well with the proposed structure. The IR spectra of compounds 2 revealed the absorption bands of $v \mathrm{NH}, \mathrm{C}=\mathrm{N}$ and v $\mathrm{S}-\mathrm{H}$; the MS showed the characteristic fragmentation pattern due to the presence of chlorine atom (Table 2).

As a point of interest, compounds $2 a, 2 b$ reacted with chloroacetic acid in glacial acetic acid acetic anhydride mixture (in adjusted temperature between $\left(40-70^{\circ} \mathrm{C}\right)$ in the presence of anhydrous sodium acetate to yield the corresponding 5, 7-diaryl-2, 3-dihydro-5H-thiazolo[3,2-a] pyrimidin-3-ones $3 a, 3 b$, respectively. The IR spectra of $3 a, 3 b$ revealed the presence of $v \mathrm{C}=\mathrm{O}$, $\mathrm{C}=\mathrm{N}$ and the absence of vNH (Table 2).

The reaction was believed to proceed via nucleophilic displacement by the sulfur nucleophile of the lactim form to the partially positive saturated carbon of the ethyl chloroacetate ester $\left(S_{N} 2\right)$, followed by internal cyclization.

Condensation of $3 a, 3 b$ with aromatic aldehydes, namely furfural, thiophene-2aldehyde, isonicotinaldehyde or benzaldehyde in the presence of $\mathrm{CH}_{3} \mathrm{COONa}$ and glacial acetic acid-acetic anhydride mixture afforded the corresponding 5, 7-diaryl2-(arylmethylene)-2, 3-dihydro-5H-thiazolo [3,2-a] pyrimidin-3-ones (4a-f). The reaction of $4 a, 4 d$ with hydroxylamine hydrochloride in refluxing glacial acetic acid containing anhydrous sodium acetate yielded the corresponding3,6,8-triaryl-2,3dihydro- $8 H$-isoxazolo $\left[5^{\prime}, 4^{`}: 4,5\right]$ thiazolo $[3,2-a]$ pyrimidines $(5 a, 5 b)$. Alkylation of compound $5 b$, with 2-chloroethylmethylether gave 6-(4-bromophenyl)-2- (2methoxyethyl)- 8-phenyl-3- (2-thienyl) -2,3-dihydro- $8 H$-isoxazolo $\quad\left[5,44^{`}: 4,5\right]$ thiazolo[2,3-a]pyrimidine $(6 a)$.

The structure of 6 was supported by the mass spectrum which revealed a molecular formula $\left(\mathrm{C}_{26} \mathrm{H}_{22} \mathrm{O}_{2} \mathrm{~S}_{2} \mathrm{~N}_{3} \mathrm{Br}\right)\left(\mathrm{M}^{+}=552\right)$. The ${ }^{1} \mathrm{H}-\mathrm{NMR}$ spectrum showed one single each at $\delta 3.34 \mathrm{ppm}$ and $\delta 3.31 \mathrm{ppm}$ for the two $\mathrm{CH}-\mathrm{N}-$ cyclic protons, a signal band at $\delta 2.1 \mathrm{ppm}$ to the three protons of $\mathrm{CH}_{3}$ and two doublets near $\delta 3.41$ and $3.45 \mathrm{ppm}$ for the $\mathrm{CH}-\mathrm{CH}$ protons, and a multiplet at $\delta 7.12-8.12 \mathrm{ppm}$ for the 12 aromatic protons (Table 2).

As a point of interest, alkylation of compound $2 b$ with 2-chloroethyl methyl ether in alcoholic sodium hydroxide afforded the corresponding 2, 4-diaryl-6-(2methoxyethyl)sulphanyl-1,2-dihydropyrimidine 7.The IR spectrum of compound 7 revealed the presence of $v \mathrm{NH}, v \mathrm{C}=\mathrm{N}$ and devoid of $v \mathrm{SH}$ (Table 2). The ${ }^{1} \mathrm{H}$ NMR spectrums revealed signals for the methyl ethyl ether protons, the $\mathrm{CH}-\mathrm{CH}$ protons, the 10 aromatic protons and the $\mathrm{NH}$ proton (Table 2). In a similar manner, cyanoethylation of compounds $2 a, 2 b$ with an equimolecular amount of 
acrylonitrile in pyridine gave 3-[4-(4-substituted-phenyl)-2-phenyl-6-sulphanyl1, 2-dihydro-1-pyrimidine]propanenitrile $(8 a, 8 b)$. Due to the ambient nature of the pyrimidine-2(1H)thione derivatives $2 a, 2 b$ either the thione [A] or thiol [B] are possible ${ }^{(17)}$. The ${ }^{13} \mathrm{C}$-NMR spectrum of $2 a, 2 b$ gave signal at $\delta 185.9$ and 187.6 assignable to thiocarbonyl carbon at C-2 which explains the nucleophilic attack of the pyrimidine ring system to the methyl carbon of the nitrile. The carbon peak due to the thiol form was clearly observed for compounds $8 a, 8 b$ around $\delta 170$ (Table 2). The IR spectra of $8 a, 8 b$ revealed the presence of the $v$ $\mathrm{SH}, \mathrm{C} \equiv \mathrm{N}$ and $\mathrm{C}=\mathrm{N}$ (Table 2).

Treatment of compounds $3 a, 3 b$ with glacial acetic acid conc. $\mathrm{HCl}$ mixture affected cyclization to the corresponding 6,8-diaryl-3,4-dihydro- $2 \mathrm{H}, 6 \mathrm{H}$ pyrimido[2,1-b][1,3]-thiazin-2-ones $9 a, 9 b$, this is in agreement with the previous findings of Aly, et al. ${ }^{(18)}$, respectively. This was confirmed by elemental analysis (Table 1) and spectral data (Table 2).

Moreover, condensation of $9 a, 9 b$ with aromatic aldehydes namely benzaldehyde and/or 2-chloro-benzaldehyde afforded the corresponding 3(arylmethylene)- 6,8-diaryl-3,4-dihydro-2H,6H-pyrimido [2,1-b] [1,3]-thiazin-2ones $10 a-10 d$, the structures of which were in agreement with their spectral data (Table 2). Compounds 10 a or $10 d$ were subjected to further ring formation. Thus, the reaction of $10 a$ or $10 d$ with hydroxylamine hydrochloride in boiling glacial acetic acid in the presence of anhydrous sodium acetate yielded 3,6,8triaryl-2,3-dihydro- $4 H, 6 H$-isoxazolo $[4,5-e]$ pyrimido[2,1- $b]$ thiazine $(11 a, 11 b)$, respectively. The structure of $11 a$ was supported by its MS which revealed a molecular formula $\mathrm{C}_{26} \mathrm{H}_{20} \mathrm{O} \mathrm{SN}{ }_{3} \mathrm{Cl}\left(\mathrm{M}^{+}=457\right)$ and ${ }^{1} \mathrm{H}-\mathrm{NMR}$ spectrum which induced one single band $\delta 2.54 \mathrm{ppm}(2 \mathrm{H})$, assigned to the $\mathrm{CH}_{2}$ protons, doublet band near $\delta 3.41 \mathrm{ppm}$ for the $\mathrm{CH}$ protons, doublet band near $\delta 10.99 \mathrm{ppm}$ for the $\mathrm{NH}$ proton (exchangeable with $\mathrm{D}_{2} \mathrm{O}$ ) and a multiplet at $\delta$ 7.12-7.89 $\mathrm{ppm}$ for the 14 aromatic protons (Table 2).

Alkylation of compound 11a with 2-chloroethyl methyl ether afforded 3,6,8triaryl-2-(2-methoxyethyl)-2,3-dihydro- $4 H, 6 H$-isoxazolo[4,5-e]pyrimido [2,1-b] $[1,3]$ thiazine (12). The IR spectra of compound 12 revealed the presence of $v$ $\mathrm{C}=\mathrm{N}, \mathrm{C}-\mathrm{O}-\mathrm{N}$ (isoxazolo), agreed well with the proposed structure (Table 2).

\section{Antimicrobial activity}

The in vitro antimicrobial activity of the new derivatives 2-6, 8 and 11 against several pathogens representatives namely, Escherichia coli, Bacillus subtilis, Mycobacterium philei, Staphylococcus aureus, Aspergillus niger and Candida albicans. The disk diffusion method ${ }^{(19,20)}$ was used. Whatman No.1 filter paper disks were sterilized by autoclaving for $1 \mathrm{hr}$ at $140^{\circ} \mathrm{C}$. The sterile disks were impregnated with the tested compounds $\left(250 \mu \mathrm{g} \mathrm{mL} \mathrm{L}^{-1}\right)$. Agar plates were uniformly surface inoculated with fresh broth culture. The impregnated disks were placed on the medium suitably spaced apart and the plates were incubated at $5^{\circ} \mathrm{C}$ for $1 \mathrm{hr}$, to permit good diffusion and were then transferred to an 
incubator at $28^{\circ} \mathrm{C}$ for $24 \mathrm{hr}$. The zones of inhibition were measured. The results of antimicrobial activity tests are listed in MICs were recorded as the minimum concentration of a compound that inhibits the growth of tested microorganisms. All of the compounds tested illustrated significant antibacterial and antifungal activity when compared with reference drugs. The antibacterial assessment revealed that the compounds possess weak activities. The MIC values are generally within the range of $3.9-250 \mu \mathrm{g} / \mathrm{ml}$ against all evaluated strains.

TABLE 3. MIC values $(\mu \mathrm{g} / \mathrm{ml})$ of compounds $2-6,8$ and 11.

\begin{tabular}{|c|cccccc|}
\hline Compd. No. & $\mathbf{A}$ & $\mathbf{B}$ & $\mathbf{C}$ & $\mathbf{D}$ & $\mathbf{E}$ & $\mathbf{F}$ \\
\hline $\mathbf{2 a}$ & 250 & 15.6 & 62.5 & 125 & 250 & 250 \\
\hline $\mathbf{2 b}$ & 250 & 125 & 31.25 & 125 & 125 & 125 \\
\hline $\mathbf{3 a}$ & 125 & 125 & 125 & 125 & 62.5 & 62.5 \\
\hline $\mathbf{3 b}$ & 125 & 62.5 & 62.5 & 62.5 & 62.5 & 125 \\
\hline $\mathbf{4 a}$ & 125 & 125 & 125 & 125 & 125 & 125 \\
\hline $\mathbf{4 b}$ & 250 & 125 & 42.5 & 125 & 250 & 250 \\
\hline $\mathbf{4 c}$ & 250 & 125 & 125 & 125 & 125 & 125 \\
\hline $\mathbf{4 d}$ & 250 & 250 & 125 & 250 & 125 & 125 \\
\hline $\mathbf{4 e}$ & 250 & 250 & 250 & 250 & 250 & 250 \\
\hline $\mathbf{4 f}$ & 125 & 31.25 & 125 & 31.25 & 62.5 & 62.5 \\
\hline $\mathbf{5 a}$ & 31.25 & 250 & 125 & 125 & 62.5 & 62.5 \\
\hline $\mathbf{5 b}$ & 125 & 31.25 & 125 & 125 & 62.5 & 62.5 \\
\hline $\mathbf{6}$ & 125 & 125 & 125 & 125 & 62.5 & 125 \\
\hline $\mathbf{8 a}$ & 250 & 250 & 250 & 3.9 & 125 & 125 \\
\hline $\mathbf{8 b}$ & 250 & 250 & 250 & 62.5 & 31.25 & 62.5 \\
\hline $\mathbf{1 1 a}$ & 125 & 15.6 & 125 & 125 & 62.5 & 125 \\
\hline $\mathbf{1 1 b}$ & 125 & 125 & 125 & 125 & 125 & 125 \\
\hline Reference substance-1 & 15.60 & 15.60 & 31.25 & 31.25 & 31.25 & 250 \\
\hline Microorganism used & & -- & -- & -- & 250 & 250 \\
\hline
\end{tabular}

References substance -1: Chloramphenicol, Microorganism used; A: Escherichia coli (NRRL B3704); B: Bacillus subtiles (NRRL B-3710); C: Mycobacterium philei (isolates obtained from AlAzhar Uni. Fac. of Science); D: Staphylococcus aureus (NRRL B-767); E: Aspergillus niger (isolates obtained from Al-Azhar Uni. Fac. of Science); F: Candida albicans (isolates obtained from Al-Azhar Uni. Fac. of Science).

In comparing their MIC values with chlorampheniol, all compounds were effective against S.aureus. Compounds $3 b, 4 f, 8 b$ and especially $8 a$ showed very high activity. Compounds $2 a, 3 b, 4 f, 5 b$ and $11 a$ have shown high activity against E.coli, while compounds $2 a, 3 b, 4 f, 5 b$ and especially $11 a$ have shown 
strong activity against B.subtilis. Compounds $2 a, 2 b, 3 b, 4 b$, have shown the highest activity against $M$. philei.

The antifungal activity of the compounds was studied with two pathogenic fungi. Flucanazole has been used as a reference for inhibitory activity against fungi. All compounds showed good antifungal activity. When compared flucanazole, thirteen compounds are more active (MIC $(250 \mu \mathrm{g} / \mathrm{ml})$, and three compounds are equipotent $(250 \mu \mathrm{g} / \mathrm{ml})$ against $A$. niger and C.albicans.<smiles>[Al]c1cc([Al])[nH]c(=S)n1</smiles>

(1)<smiles>O=C(O)C1C=C([Al])NC(=S)N1</smiles>

(2a,b)<smiles>C=CCC(C)C(C)Cl</smiles>

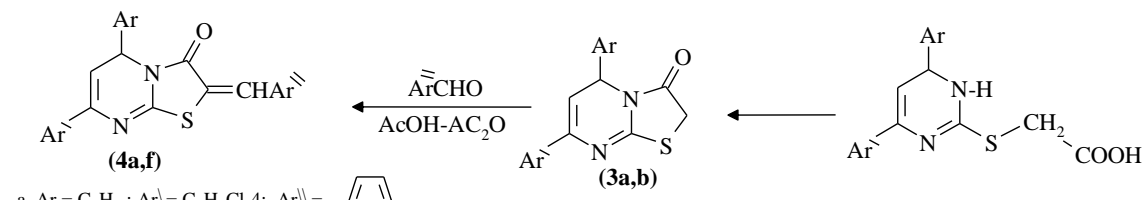

$\mathrm{a}, \mathrm{Ar}=\mathrm{C}_{6} \mathrm{H}_{5} ; \mathrm{Ar}^{\prime}=\mathrm{C}_{6} \mathrm{H}_{4} \mathrm{Cl}^{-}-4 ; \mathrm{Ar}^{\prime \prime}=$ ! I

$\mathrm{b}, \mathrm{Ar}=\mathrm{C}_{6} \mathrm{H}_{5} ; \mathrm{Ar}^{\prime}=\mathrm{C}_{6} \mathrm{H}_{4} \mathrm{Cl}-4, \mathrm{Ar}^{\prime \prime}=1$

$c, A r=\mathrm{C}_{6} \mathrm{H}_{5} ; \mathrm{Ar}^{\prime}=\mathrm{C}_{6} \mathrm{H}_{4} \mathrm{Br}-4 ; \mathrm{Ar}^{\prime \prime}=11$

$\mathrm{d}, \mathrm{Ar}=\mathrm{C}_{6} \mathrm{H}_{5} ; \mathrm{Ar}^{\prime}=\mathrm{C}_{6} \mathrm{H}_{4} \mathrm{Br}-4 ; \mathrm{Ar}^{\prime \prime}=1$

$\mathrm{e}, \mathrm{Ar}=\mathrm{C}_{6} \mathrm{H}_{5} ; \mathrm{Ar}^{\prime}=\mathrm{C}_{6} \mathrm{H}_{4} \mathrm{Br}-4 ; \mathrm{Ar}^{\prime \prime}=$

$\mathrm{f}, \mathrm{Ar}=\mathrm{C}_{6} \mathrm{H}_{5} ; \mathrm{Ar}^{\prime}=\mathrm{C}_{6} \mathrm{H}_{4} \mathrm{Br}-4 ; \mathrm{Ar}^{\prime \prime}=\mathrm{C}_{6} \mathrm{H}_{5}$

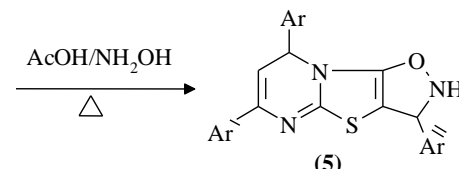

(5)

a, $\mathrm{Ar}=\mathrm{C}_{6} \mathrm{H}_{5} ; \mathrm{Ar}^{\prime}=\mathrm{C}_{6} \mathrm{H}_{4} \mathrm{Cl}-4 ; \mathrm{Ar}^{\prime \prime}=\mathrm{O}_{\mathrm{O}}$

$\mathrm{b}, \mathrm{Ar}=\mathrm{C}_{6} \mathrm{H}_{5} ; \mathrm{Ar}^{\prime}=\mathrm{C}_{6} \mathrm{H}_{4} \mathrm{Cl}-4, \mathrm{Ar}^{\prime \prime}=\left\langle{ }_{\mathrm{S}}\right.$

Scheme 1.

(6)

a, $\mathrm{Ar}=\mathrm{C}_{6} \mathrm{H}_{5} ; \mathrm{Ar}^{\prime}=\mathrm{C}_{6} \mathrm{H}_{4} \mathrm{Cl}-4, \mathrm{Ar}^{\prime \prime}=\left\langle{ }_{\mathrm{S}}\right.$

Scheme 2 .

Egypt. J. Chem. 54, No.1 (2011) 
<smiles>C[I+](C)C1=CC([Al])NC(S)=N1</smiles>

(2a,b)

$\mathrm{a}, \mathrm{Ar}=\mathrm{C}_{6} \mathrm{H}_{5} ; \mathrm{Ar}^{\prime}=\mathrm{C}_{6} \mathrm{H}_{4} \mathrm{Cl}-4$

$\mathrm{b}, \mathrm{Ar}=\mathrm{C}_{6} \mathrm{H}_{5} ; \quad \mathrm{Ar}^{\prime}=\mathrm{C}_{6} \mathrm{H}_{4} \mathrm{Cl}-4$

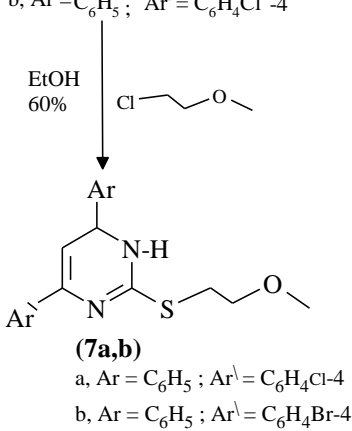

Scheme 3 .

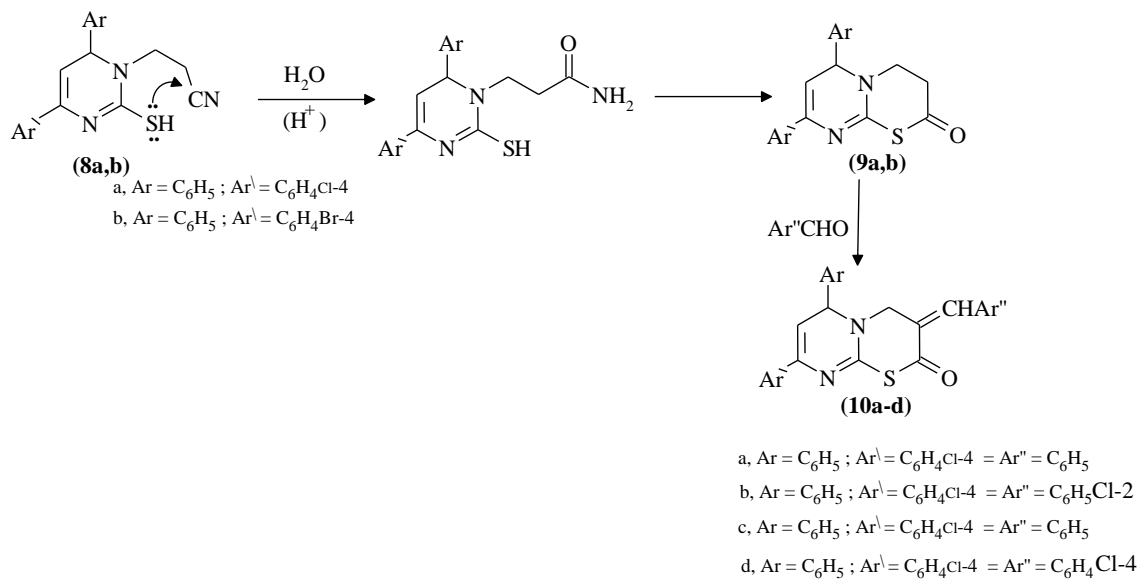

Scheme 4.

Egypt. J. Chem. 54, No.1 (2011) 


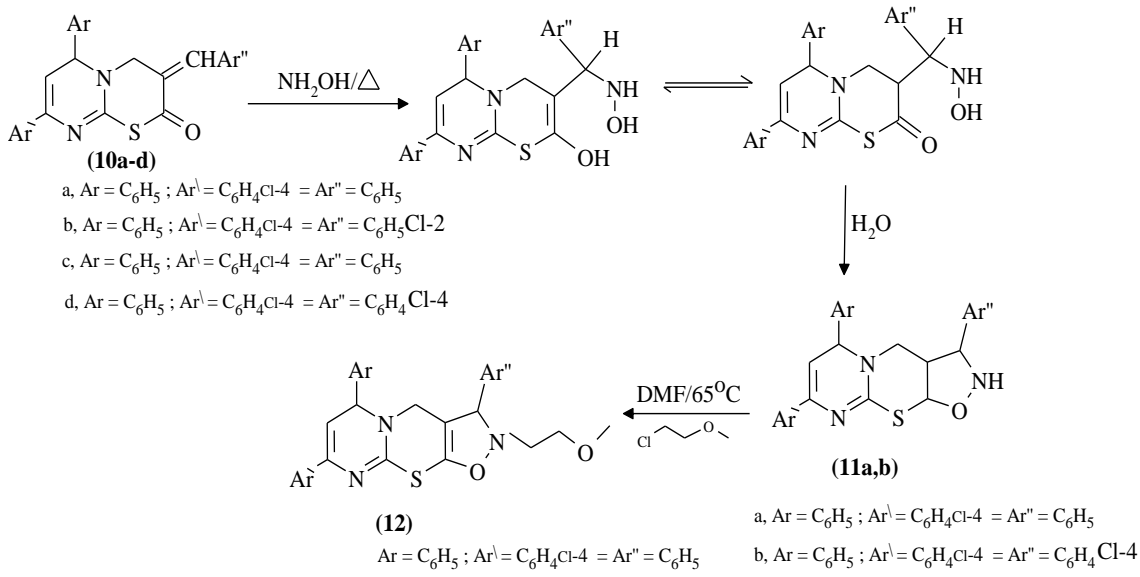

Scheme 5 .

\section{References}

1. Pecorari, P., Rinaldi, M., Costantino, L., A. Provvisionato, C. Cermelli and Portolan, M., Synthesis and biological activity of pyrimido[2,1-b][1,3]thiazine, $[1,3]$ thiazino [3,2-a] purine and [1,2,3]triazolo[4,5-d][1,3]thiazino[3,2-a]pyrimidine derivatives and thiazole analogues. Farmaco, 46, 899-911 (1991).

2. Al-Thebeiti, M.S., Synthesis of some new derivatives of thiazolo-[3, 2-a] pyrimidine3, 5, 7 (2H)-trione of potential biological activity. Boll. Chim. Farm. 140, 221-223 (2001).

3. Tozkoparan, B., Ertan, M., Kelicen, P. and Demidamar, R., Synthesis and antiinflammatory activities of some thiazolo[3,2-a]pyrimidine derivatives. Farmaco, 54, 588-593 (1999).

4. Dave, C.G., Shah, D.R., Shah, G.K., Pandya, P.S., Dave, K.C., and Patel,V.J., Synthesis and analgesic activity of 4-aminopyrido [2,3-d] pyrimidines. Indian $J$. Pharm. Sci. 48 (3) ,75-77(1986).

5. Claiborne, C.F., Critchley, S., Langston, S.P., Olhava, E.J., Peluso, S., Weatherhead, G.S., Vyskocil, S., Visiers, L., Mizutani, H. and Cullis, C., PCT Int. Appl. WO 2008/0/9124 Al, February 14, 2008, Chem. Abstr., 148, 26, 2855 (2008).

6. Zimmermann, P., Senn-Bilfinger, J., Kohl, B., Hanauer, G., Postius, S., Opferkuch, W. and Grunder, G., PCT Int. Appl. WO 98 28, 299, 2 Jul. 1998, Chem. Abstr. 129, 109095t, (1998).

7. Bos, M., Gödel, T., Riemer, C. and Sleight , A., Eur. Pat. Appl. EP 815861, 7 Jan 1998, Chem. Abstr. 128, 145382x, (1998).

Egypt. J. Chem. 54, No.1 (2011) 
8. Shigeta, S., Mori, S., Watanabe, F., Takahashi, K., Nagata, T., Koike. N., Wakayama, T. and Saneyoshi, M., Synthesis and antiherpesvirus activities of 5alkyl-2-thiopyrimidine nucleoside analogues. Antivir. Chem. Chemother. 13 (2), 67$82(2002)$.

9. Sugiura, K., Schimd, A.F., Schimd, M.M., Brown, G.F., Cancer Chemotherapy Reports part 2, 12, 3 (1), 231-308m (1972).

10. Sayed, H.H., Shamroukh, A.H. and Rashed, A.E., Synthesis and biological evaluation of some pyrimidine,pyrimido_2,1-b_1,3_thiazine and thiazolo_3,2a_pyrimidine derivatives. Acta Pharm. 56, $231-244$ (2006).

11. Solankee, A. and Patel, J., Synthesis of chalcones, pyrazolines, amino pyrimidines and pyrimidinethiones as antibacterial agents. Chem. Inform, 35 (45), (2004).

12. Gupta, S., Sachar, A., Kour, D., and Singh, J., One pot synthesis of spiro pyrimidinethiones/spiro pyrimidinones, quinazolinethiones/quinazolinones, and pyrimidopyrimidines. J. Hetero. Chem. 47 (2), 324-333 (2010).

13. Ghomi, J.S. and Ghasemzadeh, M.A., Ultrasound-assisted synthesis of dihydropyrimidine- 2-thiones. J. Serb. Chem. Soc. 76 (5), 679-684 (2011)

14. Abdel-Rahman, T.M., El-Hashash, M.A. and Soliman, F.M.A., Synthesis of some new biologically active 2,3-disubstituted quinazolin-4-ones. Boll. Chim. Farm. 137 (3), 87-92 (1998).

15. Salem, M.A.I., Madkour, H.M.F., Marzouk, M.I., Azab, M.E. and Mahmoud, N.F.H., Utility of 2-thioxopyrimidin-6-(1h)ones as ring transformer in the synthesis of fused $\mathrm{Bi}$ - and tri-cyclic heterocyclic compounds and their potential biological activities. Phosphorus, Sulfur, and Silicon and the Related Elements, 183 (10), 25962614 (2008) .

16. Abdel-Ghaffar, N.F., Kassab, R.R.S. and Soliman, F.M.A., Synthesis and reactions of new substituted pyrimidine thione derivatives as antimicrobial agents. Rev. Roum. Chim. 46 (5), 535-542 (2001).

17. Nishio, T., Fujisawa, M. and Omoto,Y., Photochemical reactions of pyrimidinethiones with alkenes . J. Chem. Soc., Perkin Trans. 1, 2523-29 (1987).

18. Aly, A.A., A facile synthesis and heteroannualation of thiazolopyrimidine and related hetero cyclic systems . Heterocycl. Chem. 45 (4), 993-998 (2008).

19. Murray, R.R., Baron, E.J., Pfaller, M.A., Tenover, F. C. and Yolken, R.H., In: G.L. Wood and J.A. Washington (Editors), Manuel of Clinical Microbiology, American Society of Microbiology, Washington, DC, 566-573 (1995).

20. NCCLS, Reference method for broth dilution antifungal susceptibility testing of yeasts approved standard, second ed., ISBN 1-56238-469-4 NCCLS Document M 27 A2 (2002).

(Received 13/7/2010;

accepted 1/6/2011)

Egypt. J. Chem. 54, No.1 (2011) 


\section{ســلوك الثيوبريميــينات تجـــاه بعــ الكواثــف الإكتروفيليــة

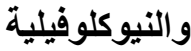

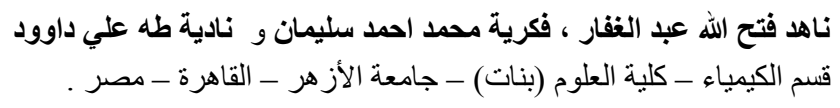

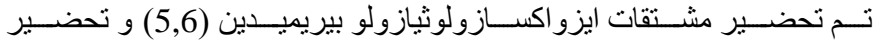

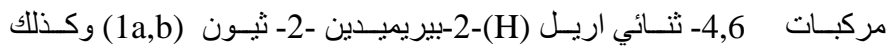

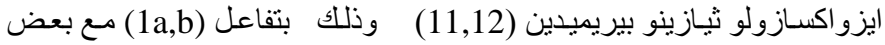

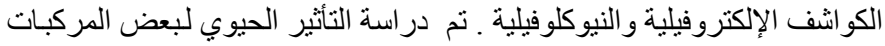

التي تم تحضير ها تجاه الميكروبات. 